\title{
Integrative assessment of brain and bone invasion in meningioma patients
}

\author{
Kerstin Zwirner ${ }^{1}$, Frank Paulsen ${ }^{1 *}$ (D), Jens Schittenhelm ${ }^{2,6,7}$, Irina Gepfner-Tuma ${ }^{3,6,7}$, Ghazaleh Tabatabai ${ }^{3,6,7}$, \\ Felix Behling ${ }^{4,6,7}$, Marco Skardelly 4,6,7 Benjamin Bender ${ }^{5,6,7}$, Daniel Zips ${ }^{1,6,7}$ and Franziska Eckert ${ }^{1,6,7}$
}

\begin{abstract}
Background: Various prognostic factors have been suggested in meningioma patients including WHO grading, brain invasion and bone involvement, for instance. Brain invasion was included as an independent criterion in the recent WHO classification. However, assessability of brain or bone involvement is often limited or varies between histopathologic, operative and imaging reports. Objective of our study was to investigate prognostic values including brain and bone involvement according to different clinical approaches.

Methods: A cohort of 111 patients was treated with primary, adjuvant or salvage irradiation between 2008 and 2017 using intensity-modulated radiotherapy. Positron-emission tomography (PET) was available for treatment planning in $81 \%$ of patients. Clinical data were extracted from the medical reports. Brain and bone involvement were stratified separately according to histopathologic, operative and imaging reports as well as judged in synopsis.

Results: WHO grade I tumours, lower estimated proliferation index, primary versus recurrence treatment and localization (i.e. skull base, optic nerve sheath) were beneficial prognostic factors for local control. Judgement of brain and bone invasion partly differed between diagnostic modalities. In synopsis, brain or bone invasion did not show a significant influence on local control rates.

Conclusions: Several previously described prognostic factors could be reproduced. However, partly divergent histopathological, surgical and image-based judgements could be found in regard to brain and bone invasion and all methods imply limitations. Therefore, we suggest a particular, complemental synopsis judgement. In synopsis, brain or bone involvement did not coherently impair local control in our irradiated patients. This might be explained by elaborate radiation techniques and PET-based treatment planning.
\end{abstract}

Keywords: Radiotherapy, Meningioma, Brain invasion, Bone involvement, Prognostic factors

\section{Background}

Primary radiotherapy or surgical resection are considered as treatment options for patients with progressive or symptomatic meningiomas [1]. Furthermore, postoperative radiotherapy is indicated if patients are at risk for local failure. Previously described risk factors for recurrence imply incomplete resection [2], higher histopathological grading according to the World Health Organization (WHO) classification [3], increased proliferation activity (e.g. Molecular Immunology Borstel (MIB-1) immunohistochemistry) [4], bone involvement [5] and brain invasion [6]. Histological

\footnotetext{
* Correspondence: Frank.Paulsen@uni-tuebingen.de

'Department of Radiation Oncology, University Hospital Tuebingen,

Hoppe-Seyler-Str. 3, 72076 Tuebingen, Germany

Full list of author information is available at the end of the article
}

brain invasion is even considered as an individual criterion for atypia (WHO grade II) and was included in the recent WHO classification 2016 [7]. Involvement of brain tissue is commonly defined by histopathological criteria referred to Perry et al. (1997) [6]. However, in this study, the majority of meningioma specimens lacked central nervous system (CNS) tissue and therefore, brain invasion could not be determined. The lack of brain tissue might be due to partial resections or because the arachnoid layer was respected by the meningioma and a resection of brain tissue was not considered necessary during surgery. The authors considered these samples (85\%) as (pathologically) 'unassessable' [6]. In regard to the 'assessable' (i.e. adjacent CNS tissue; invasion determined as present or absent) specimens, a considerable discrepancy between surgeon's gross impression

(C) The Author(s). 2019 Open Access This article is distributed under the terms of the Creative Commons Attribution 4.0 International License (http://creativecommons.org/licenses/by/4.0/), which permits unrestricted use, distribution, and reproduction in any medium, provided you give appropriate credit to the original author(s) and the source, provide a link to the Creative Commons license, and indicate if changes were made. The Creative Commons Public Domain Dedication waiver (http://creativecommons.org/publicdomain/zero/1.0/) applies to the data made available in this article, unless otherwise stated. 
and histologic findings was claimed $(\kappa=0.09$; poor agreement) [6]. Therefore, clinicopathological judgement of brain invasion implies several pitfalls. Furthermore, many risk factors were mainly established in surgically treated cohorts and translation to irradiated patients remains controversial. Even in surgically treated patients, the stand-alone impact of brain invasion on prognosis has been critically addressed, recently [8-10]. Also, judgement of bone involvement is sometimes ambiguous, even though it has considerable implications on target volume definition in radiation treatment planning [11].

The aim of our study was to validate previously described prognostic factors in patients treated with radiotherapy. Furthermore, we evaluated histopathological, operative and imaging reports individually and in synopsis in regard to brain and bone invasion to consider potential discrepancies and limitations of the individual modalities.

\section{Methods}

In our single-centre cohort of meningioma patients, we retrospectively screened for patients treated with intensity-modulated radiotherapy (IMRT) with or without prior surgery between 2008 and 2017. The analysis was approved by the local ethics committee (417/2017B02). Patients without evaluable follow-up or re-irradiated patients were excluded. Clinical features and follow-up data were extracted from the medical reports.

The extent of resection (subtotal versus gross tumour resection) was documented according to the operative report and post-operative imaging. The localization of meningiomas was subclassified into optic nerve sheath and skull base meningiomas versus 'other' meningiomas. Furthermore, the availability of positron-emission tomography (PET) with somatostatin-analogue tracers (combined with computed tomography (PET/CT) or magnet resonance imaging (PET/MRI)) for treatment planning and the intention of irradiation (primary radiotherapy, adjuvant radiotherapy, adjuvant radiotherapy after salvage surgery or salvage radiotherapy) were recorded. Salvage therapies were defined as therapy initiation for tumour recurrence or progression.

All patients in our series were treated with IMRT and image guidance as fractionated stereotactic radiotherapy. The applied radiation dose ranged between 54.0Gy (WHO grade I meningiomas, optic nerve sheath and skull base meningiomas without prior surgery) and 60.0Gy (WHO grade II meningiomas in other localizations). In case of meningiomas with close proximity to the optic nerve or chiasm, patients were treated with a simultaneous integrated boost (1.7Gy / 1.8Gy) up to a total dose of 51.0Gy / 54.0Gy (planning target volumes PTV51 / PTV54). The gross tumour volume (GTV) was defined as the contrastenhancing region in T1-weighted MRI in synopsis with the PET dataset, both co-registered to the planning CT.
An obvious dural tail was included within the GTV. For skull base meningiomas near the visual pathway or the brain stem, the PTV was defined as GTV plus a safety margin: $5 \mathrm{~mm}$ margin for the PTV51 and $2 \mathrm{~mm}$ margin for the PTV54. For other tumours, larger margins of approximately $10 \mathrm{~mm}$ alongside the meninges were used for the clinical target volume (CTV) and $5 \mathrm{~mm}$ safety margin to the PTV. In case of brain or bone involvement, approximately $10 \mathrm{~mm}$ around the cavity into the brain / bone or around the GTV were included as also described as accepted treatment strategies in recent reports $[12,13]$.

Most histopathological reports for grading were provided before the recent 2016 WHO classification was established. However, all samples with histologically proven brain invasion (which was recorded independently) from this time period were also classified as WHO grade II meningiomas because further criteria of atypia (mitotic activity) were apparent. Thus, all samples are consistent with the latest WHO classification (2016). The majority of histopathologic reports provided estimated MIB-1 staining to indicate the proliferative activity. The average values of the reports were documented and patients were arbitrarily separated into two risk groups according to the median (MIB- $1<4 \%$ and MIB$1 \geq 4 \%$ ) based on previous reports [14].

Brain and bone invasion were retrospectively assessed based on histopathological, operative and diagnostic imaging reports separately and in synopsis. Assessable data of the different modalities that allowed a distinct consideration ('yes' or 'no') varied and are shown in the Additional file 1: Figures S1 and S2. The graduation of Perry et al. (1997) [6] was used to record histopathological brain invasion. If no CNS parenchyma was reported, the sample was regarded as histopathologically unassessable. In case of adjacent CNS tissue, brain invasion was considered as either present or absent.

For the synopsis evaluation, all information was considered (Fig. 1). If data of one modality was missing and others commented on invasion, the available information was used to compensate the missing ones. In case of contradicting information, plausibility was considered by the explicitness of the reports. If there were no distinct arguments to decide otherwise, pathologic reports were ranked as most important, followed by the surgeon's description. In synopsis, invasion was considered as absent if none of the reports commented on involvement. Therefore, in synopsis evaluation, all patients of the cohort were included.

Progression-free survival (PFS) was calculated from the first day of radiotherapy and defined by local control of the irradiated lesion according to the last follow-up. For the statistical analysis we used SPSS (version 25, IBM Corp., Armonk, NY). To estimate local recurrence, the Kaplan-Meier method was applied. For comparison 


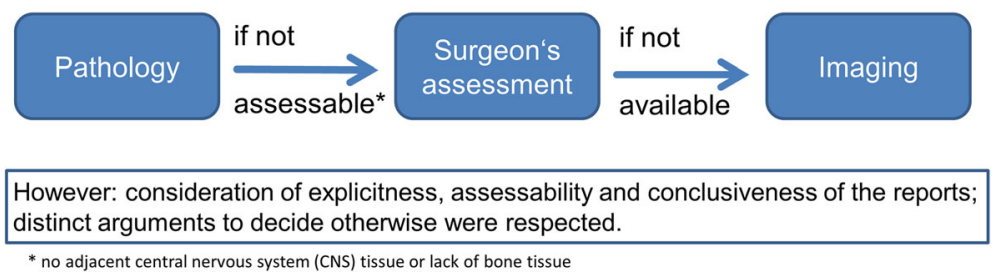

Fig. 1 Synopsis evaluation considerations

of different risk groups, we used the log-rank test. Cox regression was used for multivariate analysis and the chi-square test was performed for additional estimations. $P$-values $<0.05$ were considered significant. Cross-classification tables were used for modality comparison if numbers were considered sufficient and relevant to present interrelationships.

\section{Results}

In total, 111 patients (78 female, 33 male patients) met the inclusion criteria and were eligible for evaluation. Median age at primary diagnosis was 52 years (range 21-82 years) and at initiation of radiotherapy, the median age was 56 years (range $22-82$ years). Detailed patient and treatment characteristics are shown in Table 1 . In $81 \%$ of patients, radiotherapy planning was supported by PET/CT or PET/MRI imaging. Median follow-up was 3.3 years (range 0.3-10.2 years) and the 2year and 4-year local control rates were 97.6 and $88.7 \%$.

In our irradiated cohort, in univariate analysis we found significant differences of PFS stratified by WHO grade I versus WHO grade II meningiomas $(p=0.026)$, estimated MIB-1 staining below 4\% $(p=0.019)$, primary or adjuvant radiotherapy versus salvage treatment $(p=$ 0.003 ) and the localization (in favour of skull base and optic nerve sheath meningiomas compared to other localizations, $p=0.007)$. The respective Kaplan-Meier curves are visualized in Fig. 2. In multivariate analysis, none of the univariate prognostic factors remained significant (data not shown). We did not find significant differences in PFS regarding the resection status (subtotal versus gross total resection, $p=0.184$, data not shown).

Kaplan-Meier plots in regard to brain invasion are shown in Fig. 3. Histopathological, surgical and imagebased investigations are illustrated separately. Respective numbers of assessable reports are presented in the Additional file 1: Figure S1. The synopsis $(n=111)$ was plotted individually for clarification.
Patients with histopathologically proven brain invasion $(n=9)$ showed a trend towards improved local control compared to patients without brain invasion $(n=12)$ (Fig. 3a, $p=0.062$ ). Considering these subgroups, the patients with histologically proven brain invasive meningiomas were more likely to receive adjuvant radiotherapy $(n=6)$ rather then salvage treatment $(n=3)$. In contrast, patients without brain invasion in histopathology mainly received salvage radiotherapy $(n=7)$ rather then adjuvant treatment $(n=3)$ or adjuvant treatment after salvage surgery $(n=2)$.

In seven patients, brain invasion was explicitly described in the operative report, but no CNS tissue was found according to the histopathology report. In cases

Table 1 Patient and treatment characteristics

\begin{tabular}{lll}
\hline WHO grading ( $n$, \%) & & \\
WHO grade I & 34 & $31 \%$ \\
WHO grade II & 28 & $25 \%$ \\
Unknown & 49 & $44 \%$ \\
Initial treatment ( $n$ \%) & & \\
Primary radiotherapy & 49 & $44 \%$ \\
Initial resection, adjuvant radiotherapy & 15 & $14 \%$ \\
Salvage re-resection, adjuvant radiotherapy & 9 & $8 \%$ \\
Salvage radiotherapy & 38 & $34 \%$ \\
Resection status ( $n$, \%) & & \\
Gross total resection & 29 & $26 \%$ \\
Subtotal resection & 26 & $23 \%$ \\
Unknown & 7 & $6 \%$ \\
Radiotherapy planning ( $n$ \%) & & \\
PET-based treatment & 90 & $81 \%$ \\
No PET available & 21 & $19 \%$ \\
Localization ( $n$, \%) & & \\
Optic nerve sheath / skull base meningiomas & 72 & $65 \%$ \\
Others & 39 & $35 \%$ \\
\hline Ab & &
\end{tabular}

Abbreviations: WHO World Health Organization, PET positron emission tomography 

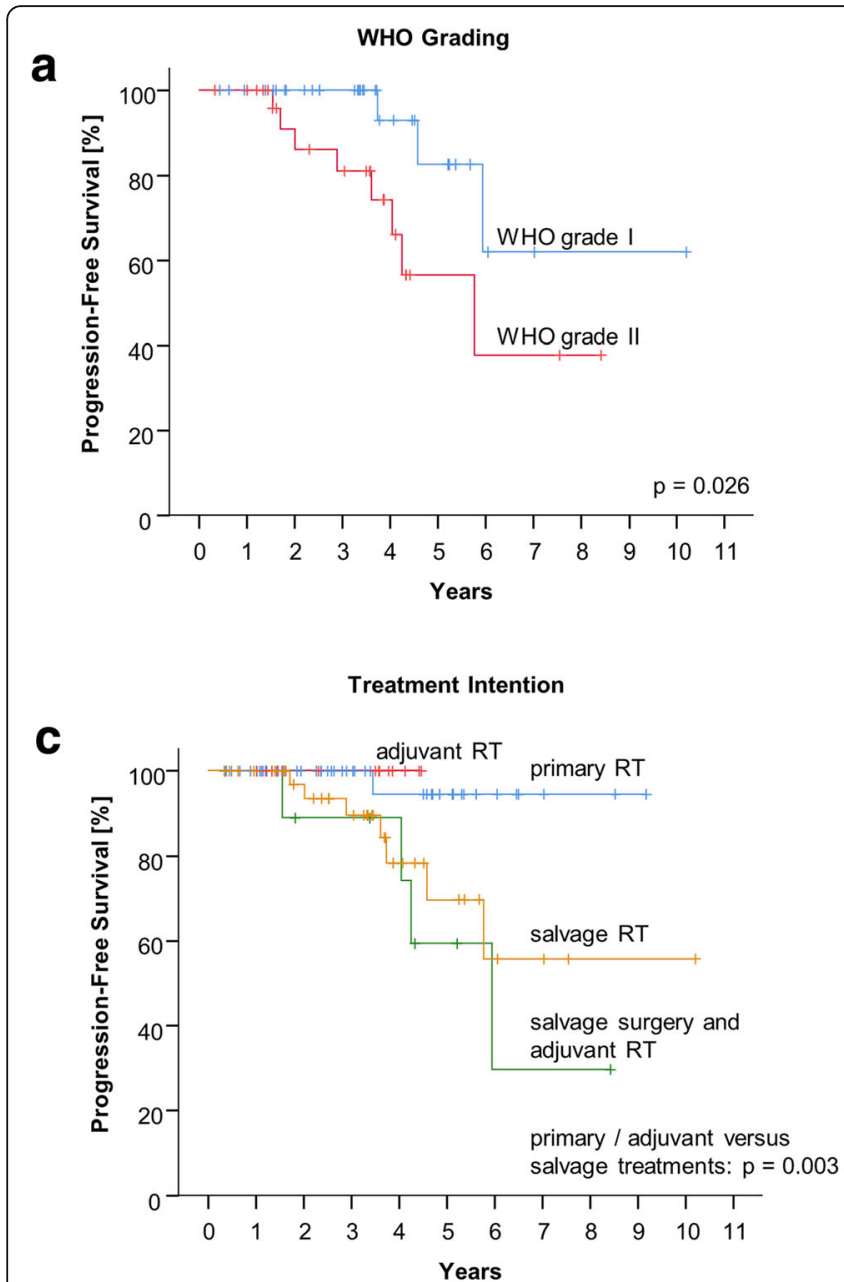

b
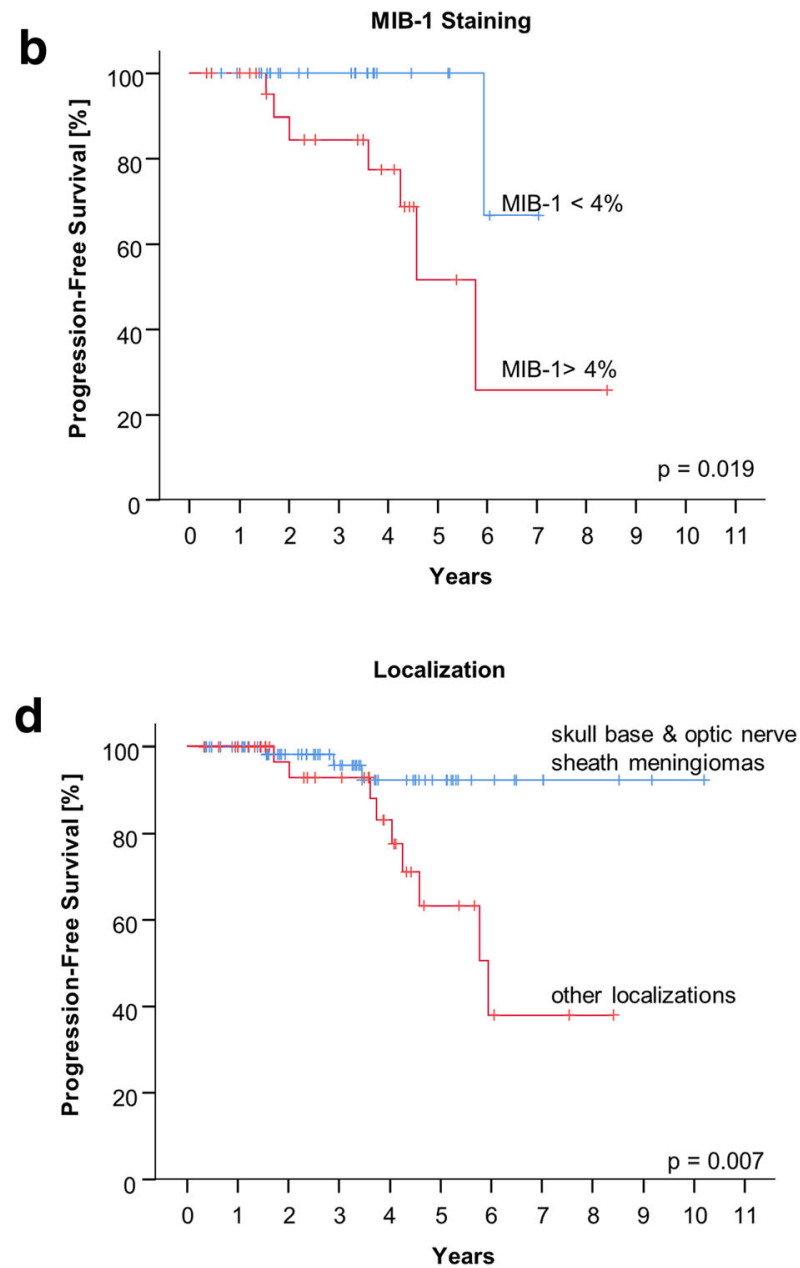

Fig. 2 Kaplan-Meier curves for progression-free survival in regard to a) World Health Organisation (WHO) grading; b) Molecular Immunology Borstel (MIB-1) immunohistochemistry (groups were separated by the median); c) intention of radiotherapy (RT) treatment (primary radiotherapy vs. adjuvant radiotherapy vs. salvage surgery and subsequent adjuvant radiation vs. salvage radiotherapy; the $p$-value is related to the primary and adjuvant versus the salvage groups); d) localization (skull base and optic nerve sheath meningiomas versus other locations)

where both reports were available, the surgeon's description of brain invasion was considerably discrepant to histopathologic findings as shown in Fig. $3 \mathrm{a} / \mathrm{b}$ and the cross-classification table (Additional file 1: Figure S3a, $p=0.205)$. If brain invasion was explicitly considered absent in operative $(n=6)$ or imaging reports $(n=16)$, no event was recorded during follow-up (Fig. 3b/c). In synopsis of the different investigation modalities, brain invasion had no significant influence on PFS in our irradiated cohort (Fig. 3d, $p=0.471$ ).

Bone invasion was suspected in 22 patients according to the operative reports but bone tissue was only found in ten histopathologic samples. In eight specimens, bone invasion was confirmed. In one patient bone invasion was suspected by the surgeon but not confirmed histopathologically, and in one case, the operative report is missing but bone involvement was reported in histopathology. Of the patients with pathologically proven bone invasion, only one received adjuvant radiotherapy whilst eight patients were irradiated by means of salvage treatment for recurrence.

The surgical and imaging assessment of bone invasion were concordant in the majority of patients. In 25 patients both reports were assessable. In these, 22 findings were concordant and three were discrepant (Additional file 1: Figure S3b, $p=0.001$ ).

Figure 4 shows the PFS curves of pathological, surgical and image-based considerations of bone invasion and the synopsis judgement. The patient with negative pathologic report considering bone invasion had tumour recurrence, whilst only one of the pathologically proven bone invasive meningiomas recurred after radiotherapy (Fig. 4a; $p$-value not estimated due to small numbers). Operative and image-based findings did not show 


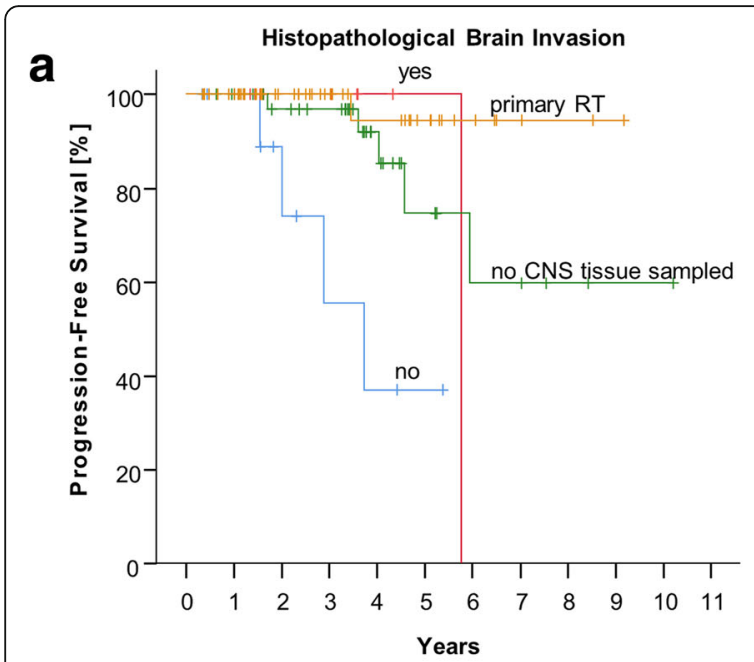

b
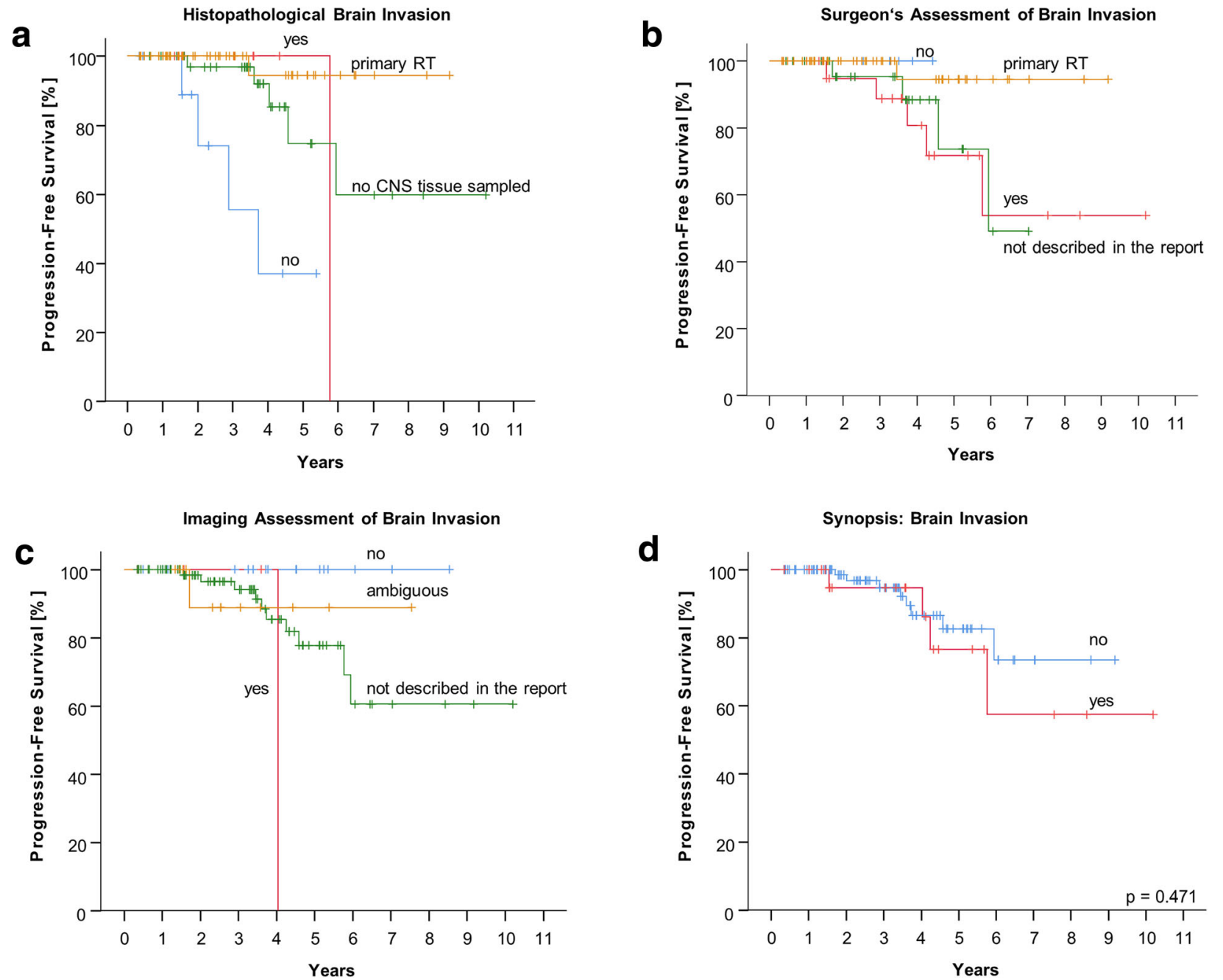

Fig. 3 Progression-free survival curves for brain invasion according to a) the histopathologic report; $\mathbf{b}$ ) the operative report; c) imaging reports and $\mathbf{d}$ ) in synopsis evaluation

significantly different PFS regarding the groups with suspected versus non-suspected bone invasion (Fig. 4b, $p=0.207$ and $4 \mathrm{c}, p=0.804)$. The synopsis consideration also did not differentiate prognosis according to bone invasion (Fig. $4 \mathrm{~d}, p=0.175$ ).

\section{Discussion}

In our cohort of meningioma patients treated with either primary, adjuvant or salvage radiotherapy, several previously described prognostic factors could be confirmed. WHO grading, MIB-1 proliferation activity, initial versus salvage treatment and the localization showed significant prognostic values in univariate analysis. These prognostic factors are well in line with several previous reports [15-17]. However, better prognosis of skull base and optic nerve sheath meningiomas in our study appears contrary to other publications. Convexity meningiomas were reported to perform better than skull base (or generally other) meningiomas due to superior surgical accessibility $[16,18]$. This previously proposed prognostic influence of the localization might have been partly overcome by recent surgical techniques or changes in management by elected partial, maximal-safe resections and adjuvant radiotherapy [2]. In spite of potentially diverse genetic and biogical features of skull base and convexity meningiomas, our surprisingly favourable results of skull base or optic nerve sheath meningiomas might be mainly explained by the selection bias of primary irradiated patients, and we presume our significant prognostic factors to be interrelated. As tumours with difficult accessibility are predominantly treated with primary radiotherapy, mostly benign histologic subtypes can be assumed in this subgroup. Convexity meningiomas are more likely to be resected a priori. Those patients typically receive radiotherapy only in case of risk factors or recurrence (negative selection). Therefore, in our cohort, the localization, initial 


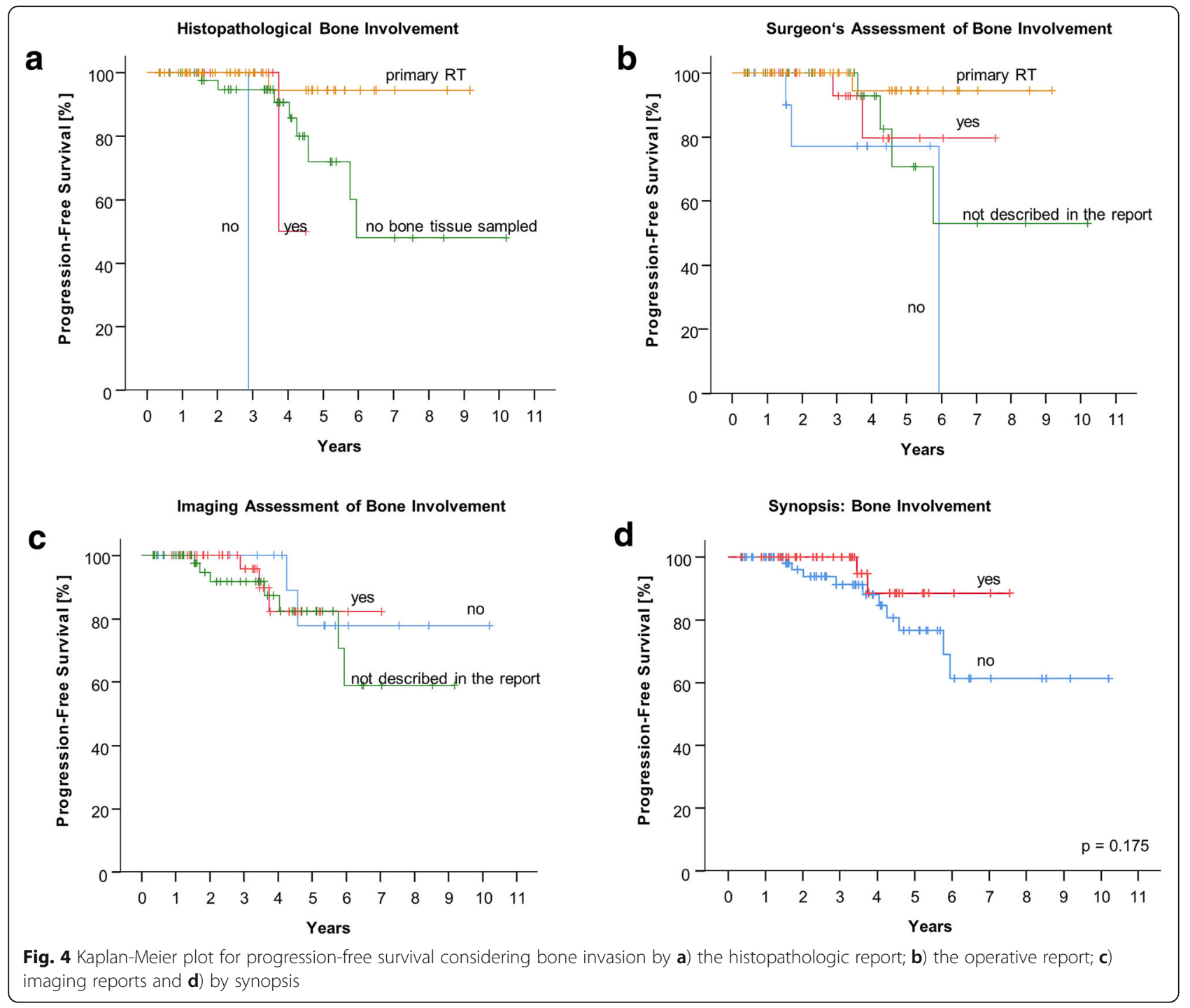

versus salvage radiotherapy, WHO grading and MIB-1 index are supposed to be related. Thus, it appears intuitive that in multivariate analysis none of the prognostic factors remained significant independently.

We decided intentionally not to re-evaluate the histopathologic or imaging reports as the therapeutic decisions in this retrospective study were based on the original reports and not on a potential re-evaluation. The intention was to retrace the daily routine setting without extensive sampling or specifically focused reports. Not all operative or imaging reports commented on brain or bone invasion in spite of potential evaluability, and relevant information could have been missed. Furthermore, MIB-1 staining was solely estimated for the routine reports by different observers, and no exact single-observer re-evaluation was conducted. These considerations might be a benefit (i.e. real setting) and limitation (i.e. potential of some incorrect assessments) of the study at the same time. Further limitations imply the retrospective character and diverse clinical constellations in regard to initial treatment and patient's performance. Furthermore, image-based consideration of brain invasion is limited to indirect hints such as massive edema or lack of invasion criteria. Besides, some subgroup analyses were limited by small numbers.

Bone or brain invasion did not arise as negative prognostic factors for PFS in synopsis of investigated aspects including pathology, surgery and imaging in our irradiated cohort. According to Perry et al. (1997), judgement of brain invasion implies several pitfalls [6]. First, in many meningioma samples, CNS parenchyma cannot be found and therefore brain invasion cannot be judged due to (intentional) surgical or pathological undersampling. In line with this concern, in several cases of our study, CNS tissue was not available for assessment. 
The sampling issues were addressed recently as extensive histopathologic sampling could improve invasion assessability [9]. Other groups did not differentiate between assessable and unassessable specimens, but diagnosed brain invasion in samples without CNS tissue as absent [10]. For pragmatic reasons this might be legitimate and probably reflects the daily practice and interpretation of reports in the clinical setting. However, by different definitions of brain invasion, the controversy of data interpretation about the prognostic value of brain invasion is even aggravated.

Sun et al. (2016) reported on patients who received adjuvant or salvage radiotherapy for meningiomas [19]. Brain invasion was found to be a negative prognostic factor for PFS in both assessable samples and unassessable samples, if those were considered as negative. In contrast, our data indicate that even in assessable samples, the predictive value of brain invasion might be limited. Histopathologically proven brain invasion showed even a trend towards improved PFS compared to patients where brain invasion was explicitly absent. This might be explained by the relevant rate of adjuvant radiotherapy treatment in our patients with histopathologically confirmed brain invasion. This intensified treatment at primary diagnosis could have prevented tumour progression or recurrence. However, these findings have to be interpreted with caution due to the limited patient number in the subgroup and need to be investigated in bigger cohorts.

As a second pitfall, divergent assessments of surgeons and pathologists regarding gross and microscopic findings of brain invasion have been described before and are difficult to interpret [6]. These challenges are in line with our data of relevant discrepancies between surgical findings and pathologic reports. A recent review suggests that a considerable tissue fraction might not be sent to pathology due to intraoperative suction, or it might not be assessable due to subtotal resection [20]. Therefore, the authors recommend sampling of different surface sites and to explicitly provide clinical information to the neuropathologist. This issue is replicated in our data. In seven patients brain invasion was suspected during surgery but no CNS tissue was found in histopathology.

Undersampling cannot be ruled out when using only one modality to judge brain invasion, which we tried to address via synopsis evaluation. For the synopsis we used a ranking including pathological, surgical and imagebased information and considered plausibility and timerelation to radiotherapy. Patients without any reliable information about brain invasion were considered as non-invaded. Therefore, all 111 patients could be classified. Synopsis-based consideration of brain invasion did not show a significant influence on PFS.

Interestingly, if the surgeon or the radiologist did exclude brain invasion explicitly, no local failure was recorded in these subgroups. Even though the number of respective patients was rather small, this information might give some indication of improved PFS.

In conclusion, the investigation of brain invasion did not show conclusive results considering PFS in our cohort and might have limited prognostic value in patients undergoing radiotherapy.

Several groups reported on bone involvement as a negative prognostic factor in atypical meningiomas [5, 21, 22]. The issue of different modalities to investigate bone involvement has been addressed before. Pieper et al. (1999) found a distinct association between radiographic evidence and histological validation of involved bony areas [23]. In line with these findings, if bone involvement was described in the operative report, these findings correlated well with imaging and histopathologic evaluations in our study. The single discrepant case with negative pathology might have been due to limited tissue and difficult differentiation between invasion and tumour metaplasia. However, in a relevant number of cases, no bone tissue was assessable for histopathology as, commonly, bone tissue is not routinely sent to pathology in spite of macroscopically visible invasion. The discrepancy between surgically assessed bone involvement and lack of bone tissue in histopathology has previously been observed [5]. The authors suggested a causal relationship of increased dural cauterization and reduced surgical aggressiveness of bone treatment. As a relevant number of local recurrences in case of bone involvement was found in this surgically treated cohort (atypical meningiomas; adjuvant radiotherapy was applied in $23 \%$ of cases), the authors speculated on residual tumour cells and discussed the potential benefit of adjuvant radiotherapy including the involved bony regions. In line with these recommendations, in our irradiated patients, bone involvement did not occur as a negative prognostic factor for PFS. Of note, PET imaging has been reported as having higher sensitivity and as being able to maintain specificity compared to MRI in detecting bone invasion [24] and seems to support target volume delineation [11]. However, radiation oncologists have to be aware of the limits of PET imaging with possible overestimation of tumor volumes due to thresholding and underestimate tumor volumes in small volume disease such as dural tails due to partial volume effects. Even though imperfect registrations and resolution issues need to be considered, improved detection of bony involvement by PET imaging might have supported treatment planning and local control in our cohort.

\section{Conclusions}

Brain and bone invasion did not conclusively influence PFS in our irradiated cohort. The potentially negative prognostic impact of brain or bone invasion might have been attenuated by elaborate radiation techniques 
(IMRT) and the support of advanced diagnostic imaging (PET/CT, PET/MRI). Considerations and assessability of brain or bone invasion can differ notably between diagnostic modalities. This might be addressed by intensified communication between specialties. Furthermore, we recommend a particular integrative synopsis judgement of histopathological, surgical and imaging information as considerable implications occur if invasion is determined.

\section{Additional file}

Additional file 1: Figure S1. Overview of assessable reports ('yes' or 'no') in regard to brain invasion and available, overlapping reports of the different modalities. Figure S2. Assessable reports ('yes' or 'no') in regard to bone involvement and the overlap of the different modalities. Figure S3. Cross-classification tables for a) the surgeon's description of brain invasion and corresponding histopathologic findings and b) operative reports considering bone invasion and associated imaging-based findings. Only assessable and distinct results ('yes' and 'no') were considered. (PDF $122 \mathrm{~kb}$ )

\section{Abbreviations}

CNS: Central nervous system; CT: Computed tomography; CTV: Clinical target volume; GTV: Gross tumour volume; IMRT: Intensity-modulated radiotherapy; MIB-1: Molecular immunology borstel; MRI: Magnet resonance imaging; PET: Positron-emission tomography; PET/CT: Positron-emission tomography combined with computed tomography; PET/MRI: Positron-emission tomography combined with magnet resonance imaging; PFS: Progressionfree survival; PTV: Planning target volume; WHO: World Health Organization

\section{Acknowledgements}

We would like to thank C. Goltermann for the language editing.

\section{Authors' contributions}

KZ collected, analyzed and interpreted the data and has written the manuscript. FP supported the analysis and interpretation of the different radiation treatments and clinical implications. JS supported the pathological analysis and interpretation. MS provided the interpretation of surgical strategies and BB supported the neuro-radiologic evaluation. KZ and FE conducted the statistical analysis. FP, JS, IGT, GT, FB, MS, BB, DZ and FE supported data analysis and interpretation. All authors read and approved the final manuscript.

\section{Funding}

K. Zwirner was supported by the intramural Fortüne / PATE Program of the Medical Faculty, Eberhard Karls University of Tuebingen under Grant 2447-00 ; and F. Eckert was partly supported by the Else Kröner-Fresenius-Stiftung, Bad Homburg, Germany under Grant 2015_Kolleg.14. We acknowledge support by Deutsche Forschungsgemeinschaft and the Open Access Publishing Fund of the University of Tuebingen. None of our funding sources had any influence on the design of the study, the collection, analysis, or interpretation of data.

\section{Availability of data and materials}

The datasets analysed during the current study are available from the corresponding author on reasonable request.

\section{Ethics approval and consent to participate}

This study was approved by the local ethics committee Tuebingen, Germany (417/2017B02) and follows the principles of the Declaration of Helsinki. For this type of study formal consent is not required.

\section{Consent for publication}

Not applicable.

\section{Competing interests}

The authors declare that they have no competing interests.

\section{Author details}

'Department of Radiation Oncology, University Hospital Tuebingen, Hoppe-Seyler-Str. 3, 72076 Tuebingen, Germany. ${ }^{2}$ Department of Neuropathology, Institute of Pathology and Neuropathology, University Hospital Tuebingen, Calwerstr. 3, 72076 Tuebingen, Germany.

${ }^{3}$ Interdisciplinary Division of Neuro-Oncology, Departments of Neurology and Neurosurgery, University Hospital Tuebingen, Hoppe-Seyler-Str. 3, 72076 Tuebingen, Germany. ${ }^{4}$ Department of Neurosurgery, University Hospital Tuebingen, Hoppe-Seyler-Str. 3, 72076 Tuebingen, Germany. ${ }^{5}$ Diagnostic and Interventional Neuroradiology, Department of Radiology, University Hospital Tuebingen, Hoppe-Seyler-Str. 3, 72076 Tuebingen, Germany. ${ }^{6}$ Center for CNS Tumors, Comprehensive Cancer Center Tuebingen, Hoppe-Seyler-Str. 3, 72076 Tuebingen, Germany. ' German Cancer Consortium (DKTK), German Cancer Research Center (DKFZ) partner site Tuebingen, Hoppe-Seyler-Str. 3, 72076 Tuebingen, Germany.

Received: 28 March 2019 Accepted: 18 July 2019

Published online: 29 July 2019

\section{References}

1. Goldbrunner R, Minniti G, Preusser M, Jenkinson MD, Sallabanda K, Houdart E, et al. EANO guidelines for the diagnosis and treatment of meningiomas. Lancet Oncol. 2016;17(9):e383-91.

2. Gallagher MJ, Jenkinson MD, Brodbelt AR, Mills SJ, Chavredakis E. WHO grade 1 meningioma recurrence: are location and Simpson grade still relevant? Clin Neurol Neurosurg. 2016;141:117-21.

3. Lam Shin Cheung V, Kim A, Sahgal A, Das S. Meningioma recurrence rates following treatment: a systematic analysis. J Neuro-Oncol. 2018;136(2):351-61.

4. Nakasu S, Fukami T, Jito J, Nozaki K. Recurrence and regrowth of benign meningiomas. Brain Tumor Pathol. 2009;26(2):69-72.

5. Gabeau-Lacet D, Aghi M, Betensky RA, Barker FG, Loeffler JS, Louis DN. Bone involvement predicts poor outcome in atypical meningioma. J Neurosurg. 2009;111(3):464-71.

6. Perry A, Stafford SL, Scheithauer BW, Suman VJ, Lohse CM. Meningioma grading: an analysis of histologic parameters. Am J Surg Pathol. 1997;21 (12):1455-65.

7. Louis DN, Perry A, Reifenberger G, von Deimling A, Figarella-Branger D, Cavenee WK, et al. The 2016 World Health Organization classification of tumors of the central nervous system: a summary. Acta Neuropathol. 2016; 131(6):803-20

8. Baumgarten P, Gessler F, Schittenhelm J, Skardelly M, Tews DS, Senft C, et al. Brain invasion in otherwise benign meningiomas does not predict tumor recurrence. Acta Neuropathol. 2016;132(3):479-81.

9. Pizem J, Velnar T, Prestor B, Mlakar J, Popovic M. Brain invasion assessability in meningiomas is related to meningioma size and grade, and can be improved by extensive sampling of the surgically removed meningioma specimen. Clin Neuropathol. 2014;33(5):354-63.

10. Spille DC, Hess K, Sauerland C, Sanai N, Stummer W, Paulus W, et al. Brain invasion in Meningiomas: incidence and correlations with clinical variables and prognosis. World Neurosurg. 2016;93:346-54.

11. Gehler B, Paulsen F, Oksuz MO, Hauser TK, Eschmann SM, Bares R, et al, [68Ga]-DOTATOC-PET/CT for meningioma IMRT treatment planning. Radiat Oncol. 2009:4:56.

12. Rogers $L$, Zhang $P$, Vogelbaum MA, Perry A, Ashby LS, Modi JM, et al. Intermediate-risk meningioma: initial outcomes from NRG oncology RTOG 0539. J Neurosurg. 2018;129(1):35-47.

13. Zollner B, Ganswindt U, Maihofer C, Corradini S, Albert NL, Schichor C, et al. Recurrence pattern analysis after [(68)Ga]-DOTATATE-PET/CT -planned radiotherapy of high-grade meningiomas. Radiat Oncol. 2018;13(1):110.

14. Abry E, Thomassen IO, Salvesen OO, Torp SH. The significance of Ki-67/MIB1 labeling index in human meningiomas: a literature study. Pathol Res Pract. 2010;206(12):810-5.

15. Chen WC, Magill ST, Wu A, Vasudevan HN, Morin O, Aghi MK, et al. Histopathological features predictive of local control of atypical meningioma after surgery and adjuvant radiotherapy. J Neurosurg. 2018:1-8.

16. de Almeida AN, Pereira BJA, Pires Aguiar PH, Paiva WS, Cabrera HN, da Silva CC, et al. Clinical outcome, tumor recurrence, and causes of death: a longterm follow-up of surgically treated Meningiomas. World Neurosurg. 2017; 102:139-43. 
17. Chen WC, Hara J, Magill ST, Wu A, Aghi MK, Theodosopoulos PV, et al. Salvage therapy outcomes for atypical meningioma. J Neuro-Oncol. 2018; 138(2):425-33.

18. Mirimanoff RO, Dosoretz DE, Linggood RM, Ojemann RG, Martuza RL. Meningioma: analysis of recurrence and progression following neurosurgical resection. J Neurosurg. 1985;62(1):18-24.

19. Sun SQ, Cai C, Murphy RK, DeWees T, Dacey RG, Grubb RL, et al. Radiation therapy for residual or recurrent atypical meningioma: the effects of modality, timing, and tumor pathology on long-term outcomes. Neurosurgery. 2016;79(1):23-32.

20. Brokinkel B, Hess K, Mawrin C. Brain invasion in meningiomas-clinical considerations and impact of neuropathological evaluation: a systematic review. Neuro-Oncology. 2017;19(10):1298-307.

21. Nowak A, Dziedzic T, Krych P, Czernicki T, Kunert P, Marchel A. Benign versus atypical meningiomas: risk factors predicting recurrence. Neurol Neurochir Pol. 2015;49(1):1-10.

22. Shakir SI, Souhami L, Petrecca K, Mansure JJ, Singh K, Panet-Raymond V, et al. Prognostic factors for progression in atypical meningioma. J Neurosurg. 2018:1-9.

23. Pieper DR, Al-Mefty O, Hanada Y, Buechner D. Hyperostosis associated with meningioma of the cranial base: secondary changes or tumor invasion. Neurosurgery. 1999:44(4):742-6 discussion 6-7.

24. Kunz WG, Jungblut LM, Kazmierczak PM, Vettermann FJ, Bollenbacher A, Tonn JC, et al. Improved detection of Transosseous Meningiomas using (68)Ga-DOTATATE PET/CT compared with contrast-enhanced MRI. J NuC Med. 2017;58(10):1580-7.

\section{Publisher's Note}

Springer Nature remains neutral with regard to jurisdictional claims in published maps and institutional affiliations.

Ready to submit your research? Choose BMC and benefit from:

- fast, convenient online submission

- thorough peer review by experienced researchers in your field

- rapid publication on acceptance

- support for research data, including large and complex data types

- gold Open Access which fosters wider collaboration and increased citations

- maximum visibility for your research: over $100 \mathrm{M}$ website views per year

At BMC, research is always in progress.

Learn more biomedcentral.com/submissions 